Projets

de paysage

\section{Projets de paysage}

Revue scientifique sur la conception et l'aménagement de l'espace

$16 \mid 2017$

Arbres et paysages

\title{
Multivalorisation et patrimonialisation de paysages d'arbres hors forêt en Ariège
}

Multivalorisation and Heritagisation of Non-Woodland Tree Landscapes in the Region or Ariège

Sylvie Guillerme, Jean-Paul Métailié et Éric Maire

\section{(2) OpenEdition}

\section{Journals}

Édition électronique

URL : http://journals.openedition.org/paysage/5839

DOI : 10.4000/paysage.5839

ISSN : 1969-6124

Éditeur :

École nationale supérieure du paysage de Versailles-Marseille, Institut national des sciences appliquées Centre Val de Loire - École de la nature et du paysage, École nationale supérieure d'architecture et de paysage de Bordeaux, École nationale supérieure d'architecture et de paysage de Lille, Agrocampus Angers

\section{Référence électronique}

Sylvie Guillerme, Jean-Paul Métailié et Éric Maire, « Multivalorisation et patrimonialisation de paysages d'arbres hors forêt en Ariège », Projets de paysage [En ligne], 16 | 2017, mis en ligne le 11 juillet 2017 , consulté le 30 avril 2020. URL : http://journals.openedition.org/paysage/5839; DOI : https://doi.org/ $10.4000 /$ paysage.5839

Ce document a été généré automatiquement le 30 avril 2020.

Projets de paysage 


\section{Multivalorisation et patrimonialisation de paysages d'arbres hors forêt en Ariège}

Multivalorisation and Heritagisation of Non-Woodland Tree Landscapes in the Region or Ariège

Sylvie Guillerme, Jean-Paul Métailié et Éric Maire

1 En France comme ailleurs dans le monde, la gestion agricole des terres a façonné une mosaïque de paysages d'une grande diversité. Pour Galochet et Simon (2003) l'arbre peut être un indicateur historique et social témoignant de la relation que l'homme entretient avec son environnement par l'étude de la mise en valeur passée et présente de cet élément dans son milieu. Il est un "un élément incontournable du diagnostic paysager comme de l'analyse écologique, l'arbre devient un objet géographique complexe, difficile à enfermer dans une approche univoque ». Les arbres hors forêt (AHF) constituent pour une grande part l'héritage d'agrosystèmes qui ont utilisé l'arbre comme élément essentiel de leur configuration. Cette arboriculture a nettement contribué à la diversité végétale ou paysagère des territoires et a historiquement fourni une grande variété de produits agricoles. Différents auteurs estiment par exemple que les vergers de haute-tige représentent sans doute une des plus emblématiques formations d'arbres hors forêt du fait de leur grande valeur fruitière (Coulon, 2000 ; Coulon et al., 2005). En tant que composante de systèmes agraires très anciens, l'arbre hors forêt s'inscrit dans la durée. Cependant, cette permanence dans l'espace ne doit pas masquer les évolutions qui se sont produites.

2 Depuis la Seconde Guerre mondiale, ces paysages ont souvent changé. À partir des années 1960, ces systèmes arborés extrêmement diversifiés sont souvent tombés en désuétude, ont été détruits ou remplacés par une arboriculture intensive et standardisée transformant radicalement les paysages. La longévité de l'arbre peut masquer une tendance à l'abandon et bien des systèmes agroforestiers ont aujourd'hui perdu leur utilité et leurs fonctionnalités. Certains paysages d'arbres hors forêt actuels peuvent ainsi être considérés comme des reliques et on peut même parler de situation 
critique pour la survie de certains systèmes agro-sylvo-pastoraux traditionnels complexes, comme par exemple les pâturages arborés et prés-vergers.

Quelques types de paysages arborés européens, comme les grandes dehesas en Espagne, ou les bocages, sont très renommés et ont fait l'objet de nombreux travaux. Mais les paysages «ordinaires" des agro-sylvo-systèmes traditionnels, dont la qualité esthétique est moins marquée ou la productivité marginale, ont moins attiré l'attention. Pourtant, du fait de leur fréquence et de leur extension spatiale, ils représentent un patrimoine qui peut être reconnu en tant qu'héritage intergénérationnel et ressource pour l'avenir. Ils constituent un anthroposystème aux multiples enjeux reposant sur les trois piliers du développement durable : un enjeu écologique, biologique et génétique (réservoir de biodiversité) ; un enjeu économique (agropastoralisme, artisanat, éco- et agrotourisme, etc.); et enfin un enjeu socioculturel, symbolique et historique (pratiques, usages, modes de gestion) relevant de nombreux savoirs et savoir-faire. Ils représentent une des dimensions du patrimoine culturel qu'il convient de prendre en compte dans les politiques locales et régionales de développement durable (Guillerme et al., 2013). En outre, le rôle de la conservation de ces paysages pour la gestion de la biodiversité a donné lieu à l'échelle internationale à la conceptualisation du High Nature Value Farming, en particulier en ce qui concerne les paysages pastoraux (O'Rourke et Kramm, 2012). Des solutions ont commencé à être appliquées, et l'essor de systèmes agroforestiers dits "modernes", associant des feuillus pour la production de bois d'œuvre aux cultures ou aux pâturages, peut offrir de nouvelles méthodes d'intégration des arbres dans les systèmes agraires des pays européens. Mais l'évolution des paysages arborés traditionnels pose la question générale de leur conservation ou leur valorisation.

4 Quels enjeux environnementaux, socioculturels, scientifiques et politiques cette évolution traduit-elle? En s'appuyant sur l'exemple de la vallée du Salat, en Ariège, issu de travaux de recherche sur les paysages d'arbres hors forêt (Guillerme, 2010, 2014), nous verrons la perception de ces paysages, de leurs perspectives d'évolution et des enjeux liés à leur sauvegarde, à leur rénovation ou à leur renouveau.

\section{Territoire d'étude et méthodologie}

5 Le terrain d'étude de la basse vallée du Salat (figure 1) se situe dans l'ancienne région Midi-Pyrénées, dans le Bas-Couserans, à environ une heure de route au sud de Toulouse. La vallée du Salat, à cheval sur les départements de l'Ariège et de la HauteGaronne, est un territoire de transition des pré-Pyrénées, situé entre les plaines, collines et coteaux du département de l'Ariège et le début du massif des Pyrénées ariégeoises. La basse vallée du Salat se compose d'une plaine et de coteaux (figure 2) et constitue un lieu de passage entre la plaine et la montagne. L'ensemble est encadré par les hauteurs des montagnes du Couserans, qui culminent au loin à plus de $2800 \mathrm{~m}$ d'altitude. 
Figure 1. Vallée du Salat, localisation du terrain d'étude

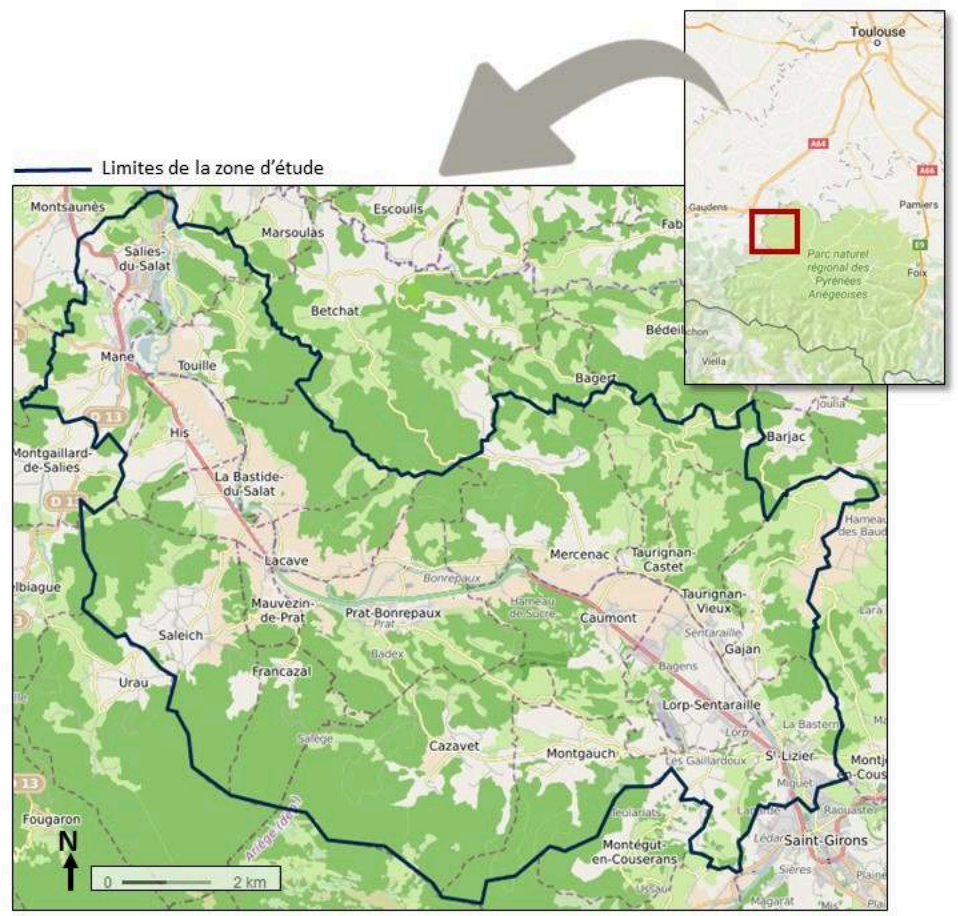

Figure 2. La basse vallée du Salat, entre plaine et coteaux

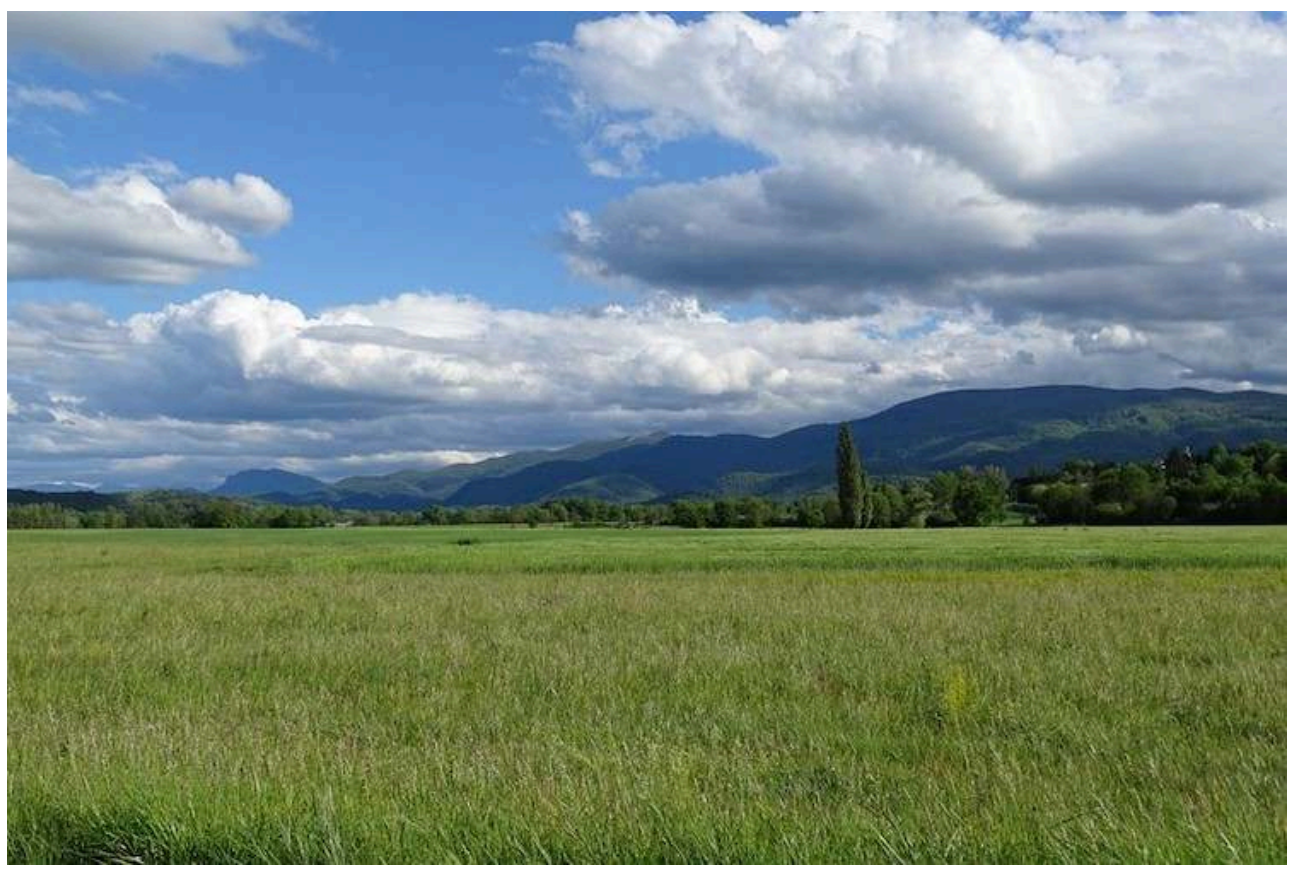

Prairies et cultures (au premier plan) sont relayées sur les marges par des coteaux très arborés. À l'arrière-plan, des sommets pyrénéens dominent le paysage.

Source : Sylvie Guillerme.

6 La plaine alluviale, sur l'ancien lit majeur de la rivière du Salat (affluent de la Garonne), d'une largeur de deux à trois kilomètres, est en majorité occupée par des cultures céréalières et des prairies bénéficiant des alluvions qui recouvrent le fond de vallée. Ici 
des paysages mixtes, où les arbres se mélangent encore à certaines cultures, ont pu se maintenir ; mais ils restent le plus souvent cantonnés dans un rôle de haie brise-vent, d'arbres isolés, ou situés en limite de parcelles de moindre intérêt agronomique. Les coteaux, aux reliefs modestes avoisinant les $500 \mathrm{~m}$ d'altitude et qui encadrent cette plaine, sont principalement dédiés à l'élevage bovin, à la polyculture et aux prairies. L'arbre y est très présent, avec des haies, des bosquets, une forte composante forestière, mais aussi de nombreuses friches qui réduisent peu à peu les clairières et illustrent un phénomène de déprise agricole et de fermeture des milieux (figure 3). Ici ou là subsistent d'anciens vergers (figure 4 ) et parfois même des vestiges de hautains (figure 5). En dehors des forêts et des bosquets, les bocages, vergers et ripisylves forment encore le maillage du paysage, mais les pratiques traditionnelles sont peu maintenues et l'enfrichement est rapide par endroits (figure 6), comme dans d'autres secteurs en Haute-Garonne et Ariège.

Figure 3. Accrus forestiers de frênes ayant envahi une ancienne prairie dans les coteaux au nord de la vallée

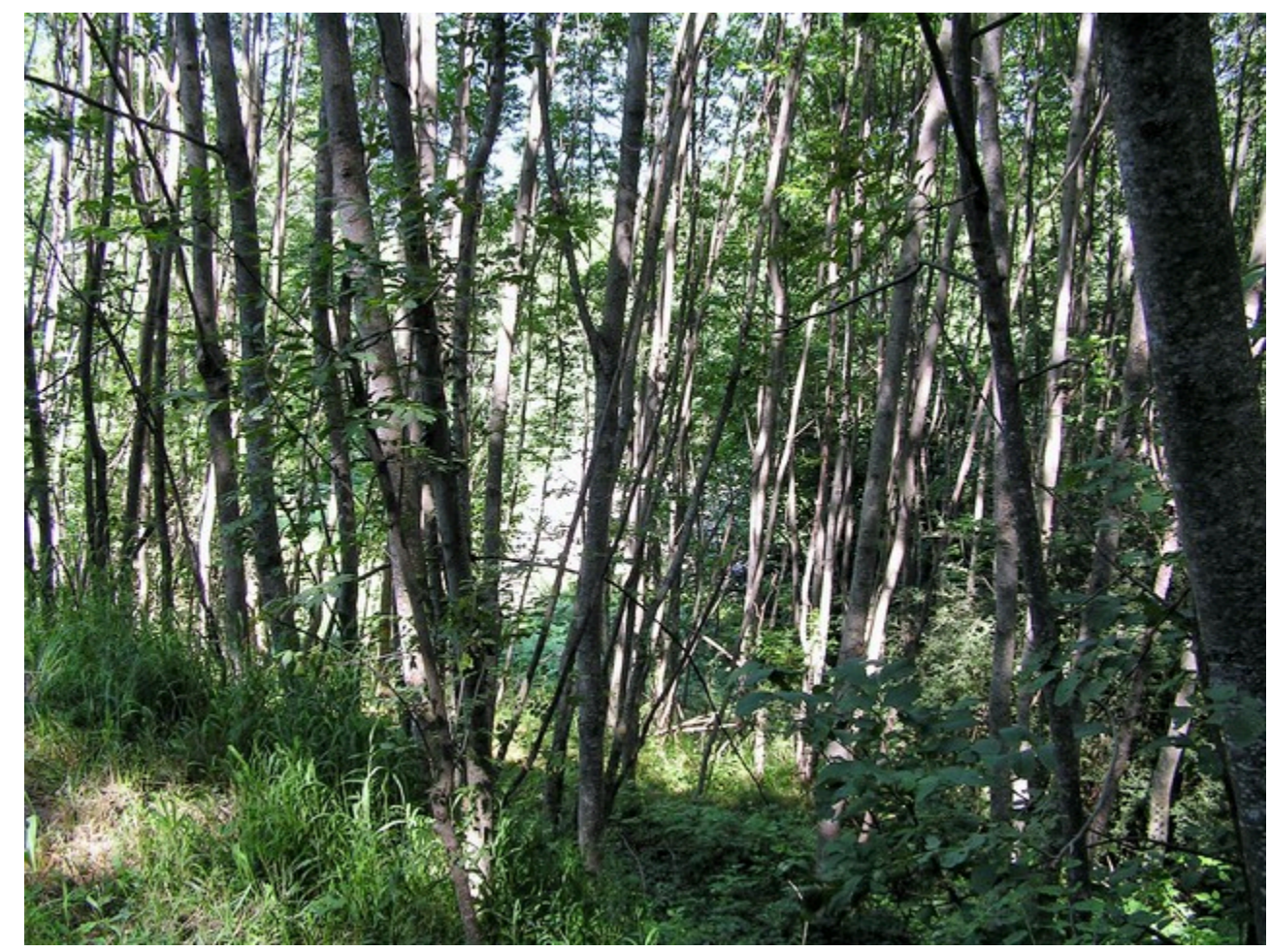

Source : Sylvie Guillerme. 
Figure 4. Ancien verger de pommiers à Saleich

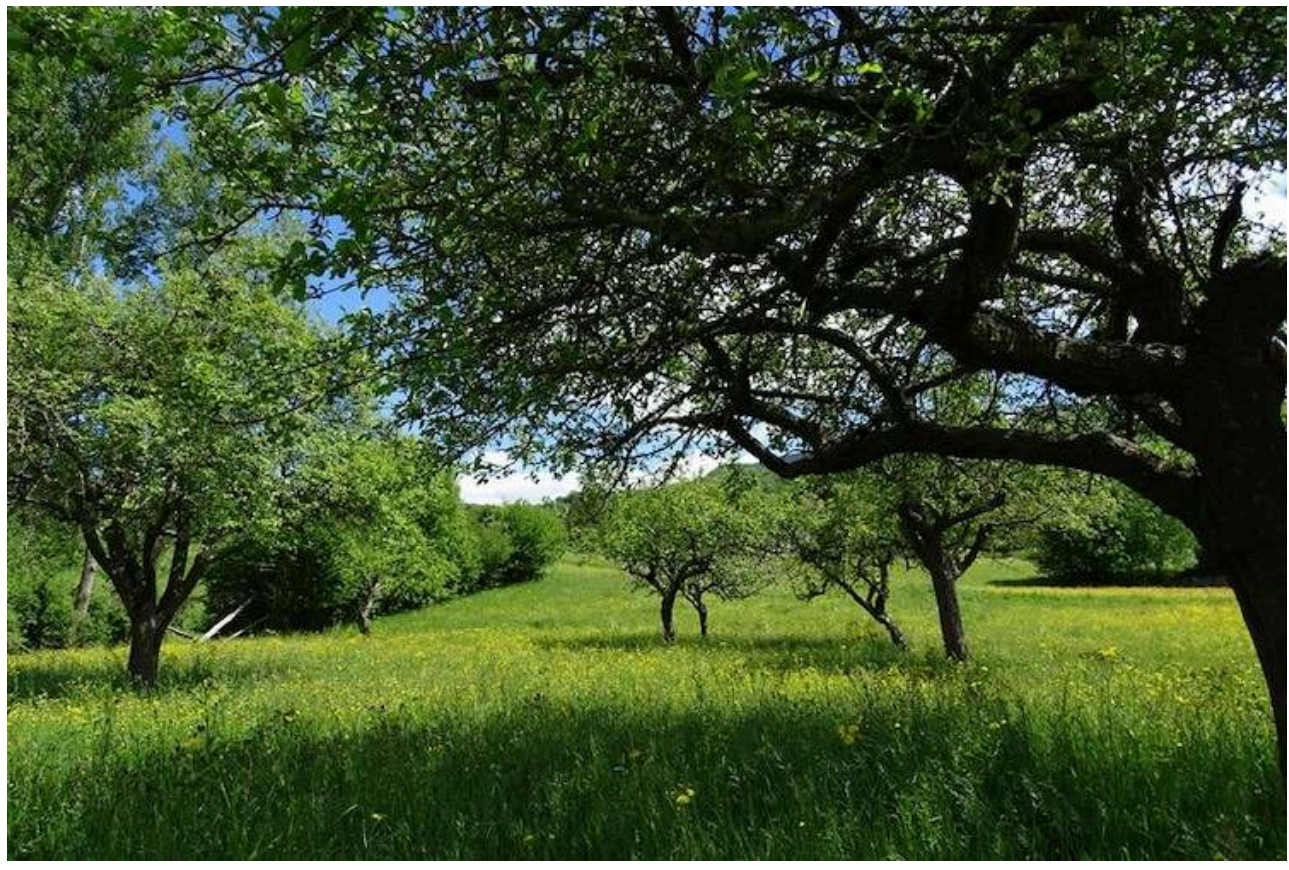

Source : Sylvie Guillerme.

Figure 5. Hautain associant vignes et saules, avec du maraîchage entre les rangs, près de PratBonrepaux

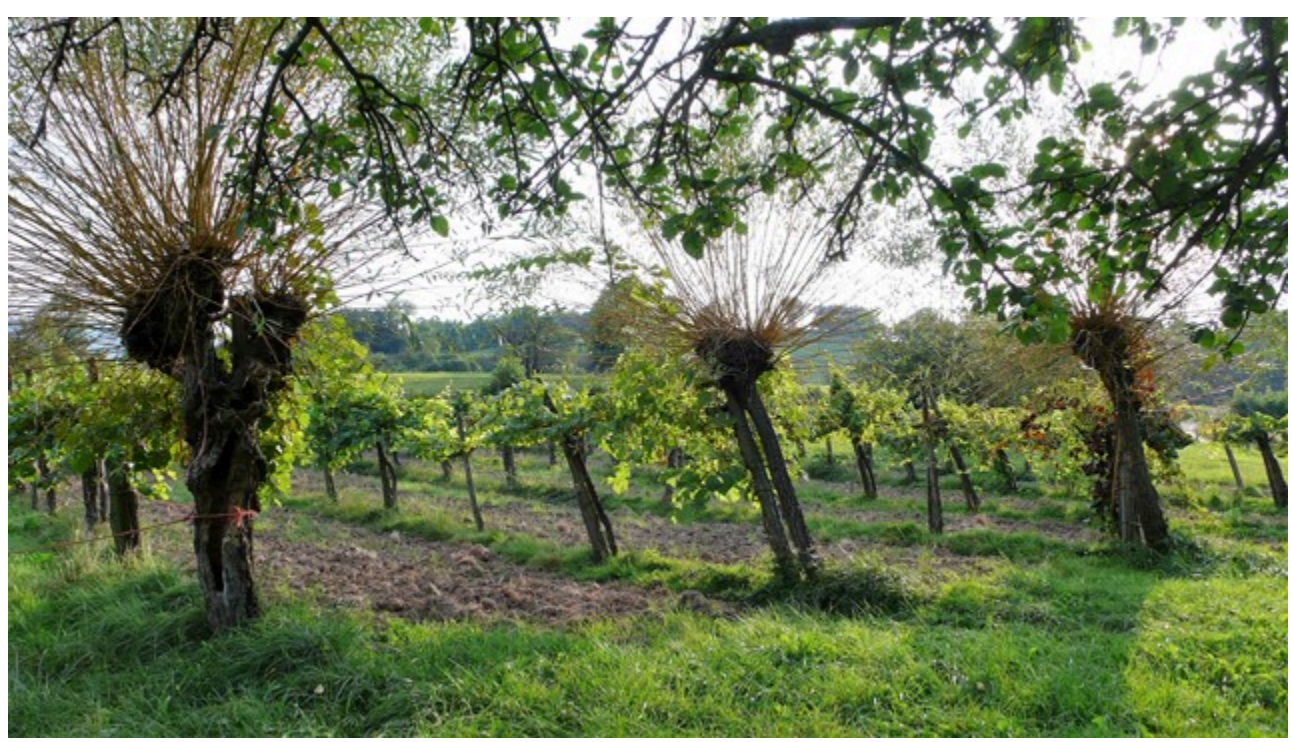

Source : Sylvie Guillerme. 
Figure 6. Fermeture progressive du paysage par embroussaillement

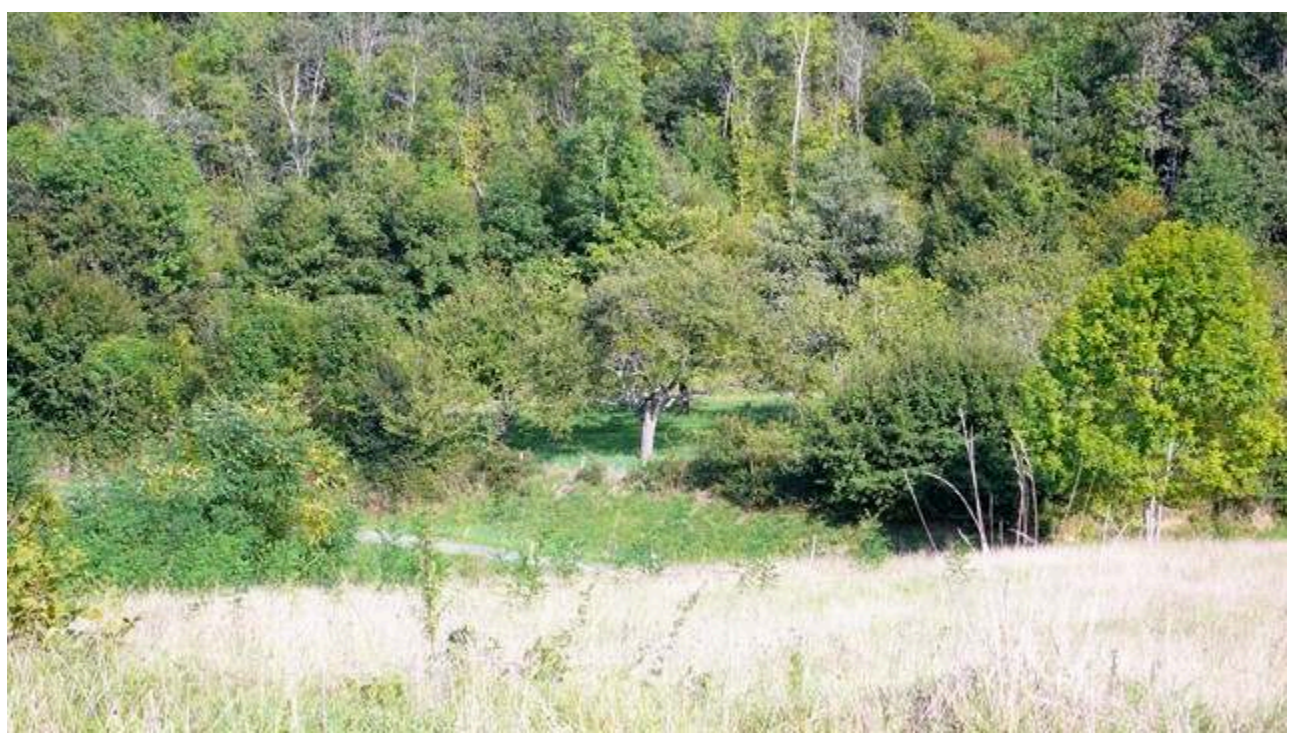

Source : Sylvie Guillerme.

7 Le maillage urbain est constitué des petites villes de Salies-du-Salat (près de 2000 habitants) et Saint-Girons (6550 habitants). Le reste du territoire est composé de petites communes (Mane, Prat-Bonrepaux, Lorp-Sentaraille...) qui forment des taches urbaines de faible envergure, ainsi que des hameaux et des petits villages parsemés. La multiplication de lotissements pavillonnaires autour des villages témoigne de l'arrivée de nouveaux habitants.

8 Sur ce terrain, peu densément peuplé, les zones naturelles ou semi-naturelles sont nombreuses : des forêts d'essences mixtes sont bien représentées sur les coteaux de la vallée, principalement au sud, et la hêtraie donne un avant-goût des paysages de montagne des Pyrénées ariégeoises. Le commerce du bois représente une part importante de l'industrie de la basse vallée du Salat. La présence d'ouvrages hydroélectriques sur la rivière témoigne d'une industrie papetière qui occupait jadis une place économique importante dans la vallée. Le Salat sert aussi de refuge à plusieurs espèces emblématiques telles que la loutre d'Europe ou le desman des Pyrénées, et son intérêt écologique l'a fait classer en site Natura 2000 en 2009. L'arbre hors forêt (AHF) est donc un élément important des paysages de ce territoire, mais la situation est contrastée: sa présence en plaine a diminué, alors que la végétation ligneuse prend de l'ampleur sur les coteaux en lien avec une fermeture des milieux.

9 Dans ce travail sur les arbres hors forêt, le paysage tel que perçu par les acteurs locaux a été central. Le concept de paysage, polysémique, y est envisagé comme "objet construit par les pratiques sociales en interaction avec des processus biophysiques " (Luginbühl, 2004), en lien avec la définition du paysage donnée par la Convention européenne du paysage qui définit celui-ci comme « une partie de territoire telle que perçue par les populations, dont le caractère résulte de l'action de facteurs naturels et/ ou humains et de leurs interrelations" (Conseil de l'Europe, 2000). L'approche méthodologique, issue de la géographie sociale, repose sur un travail d'enquêtes qualitatives. Des entretiens semi-directifs ont été menés auprès de 46 acteurs du territoire de la basse vallée du Salat (tableau 1). Quatre grandes catégories d'acteurs ont été distinguées: élus, techniciens et chargés de mission, membres d'associations, et 
agriculteurs. La diversité des points vue prévalait, et non la création de données statistiques. Aussi le panel de personnes interrogées compte aussi bien des agriculteurs engagés dans l'élevage, la polyculture, l'agriculture biologique ou conventionnelle, des retraités et des actifs; les membres d'associations sont engagés dans des structures associatives œuvrant en particulier pour la protection de l'environnement, ou le renouveau des arbres hors forêt mais aussi pour la chasse et la pêche ; et la catégorie des techniciens et chargés de mission regroupe des personnes travaillant notamment pour les chambres d'agriculture, le Parc naturel régional des Pyrénées ariégeoises et l'office national des forêts. Certains acteurs ayant une "double casquette", en particulier les élus, il leur était demandé de préciser à quelle fonction se rapportaient leurs propos. Les grands thèmes abordés étaient l'évolution des paysages, de la composante arborée et de la biodiversité, leur gestion et leurs enjeux.

Tableau 1. Acteurs enquêtés dans la basse vallée du Salat

\begin{tabular}{|l|l|}
\hline Type d'acteurs & Nombre \\
\hline Élus & 13 \\
\hline Techniciens et chargés de mission & 8 \\
\hline Membres d'associations & 10 \\
\hline Agriculteurs & 15 \\
\hline
\end{tabular}

10 Pour attester la dynamique des paysages, un travail de cartographie diachronique a en outre été conduit, à partir d'images aériennes orthorectifiées pour la période entre 1942 et 2008, et complété par des visites de terrain (Menillet, 2013). Ce travail a été enrichi par une cartographie de la trame arborée réalisée par télédétection sur le secteur de Prat-Bonrepaux (en Ariège) par classification à partir d'images Pleiades à $0,65 \mathrm{~m} /$ pixel, cette résolution favorisant l'extraction des arbres isolés et des haies (Maire et al., 2012). Les différentes données recueillies permettent de qualifier ces paysages, tant au niveau historique, qu'économique, écologique ou patrimonial.

\section{Des paysages arborés qui ont évolué}

11 De nombreux témoignages recueillis attestent une réalité économique, culturelle et paysagère des arbres hors forêt, et notamment de l'arboriculture fruitière constituée en vergers dans la basse vallée du Salat, comme dans tout le piémont pyrénéen. Ces vergers traditionnels de haute-tige dans un premier temps utilisés pour la consommation familiale, ont progressivement constitué une activité économique d'importance au cours de la première moitié $d u x^{e}$ siècle. Les arbres étaient généralement dispersés dans les champs, sur les talus et dans les haies, ou encore le long des chemins et des routes. Avec l'augmentation de la demande en fruits, les propriétaires des vergers ont adopté de nouvelles méthodes, et les variétés locales ont peu à peu été délaissées pour des variétés plus productives ou aux caractéristiques plus propices à la vente. Les vergers de haute-tige ont alors progressivement été laissés à l'abandon ou arrachés et les savoirs et savoir-faire ont cessé d'être transmis. 
12 La polyculture vivrière occupait la place centrale dans l'exploitation agricole, et les surplus étaient vendus. La modernisation de l'agriculture et les programmes politiques et financiers associés des années de l'après-guerre ont entraîné une recomposition du paysage agricole. Le maiis et l'élevage ont pris beaucoup d'ampleur, alors que les haies du bocage ont progressivement été arrachées ou abandonnées. La politique agricole commune a joué un rôle prépondérant dans l'augmentation des parcelles en maïsiculture et de l'élevage, ainsi que dans l'«introduction des maïs hybrides américains à haut rendement " dès les années 1960. Dans le piémont pyrénéen la transition a été plus progressive qu'ailleurs mais a quand même été réalisée : « En 10, 20 ans, on a mis des produits chimiques, on a coupé les arbres dans les prés-vergers parce qu'ils gênaient les tracteurs, on a arraché les haies.» (Représentante d'association de restauration de vergers.)

13 Cette évolution des pratiques s'est accompagnée de changements de valeurs. La société a évolué et, face à ce "modernisme ", ce qui est hérité du passé, des "anciens ", est devenu obsolète et a perdu son intérêt faute de rentabilité économique. C'est notamment le cas des vergers de haute-tige, peu productifs et des arbres hors forêt en général. Ces derniers ont progressivement été arrachés ou laissés à l'abandon, sans entretien. Peu à peu, en quelques dizaines d'années, dans la vallée du Salat comme dans le Couserans et le Comminges voisin, les vergers de plein-vent qui étaient une importante activité de production et de vente ont disparu. Cette évolution de la composante arborée dans les paysages est soulignée dans les entretiens : « il y en avait partout ", mais à l'heure actuelle il n'y en a plus ou ce ne sont que des « reliques ».

14 Cette évolution des paysages est aussi attestée par un travail de cartographie diachronique. On constate sur la figure 7 , sur un pas de temps de près de cinquante ans (entre 1942 et 2008, sur un secteur de la commune de Prat-Bonrepaux), non seulement un enfrichement et l'augmentation des surfaces boisées mais également l'accroissement des arbres d'ornement et la diminution des vergers. 
Figure 7. Évolution de l'occupation du sol de la composante arborée et arbustive dans la basse vallée du Salat, secteur de Prat-Bonrepaux (Ariège) en près de cinquante ans, entre 1942 et 2008

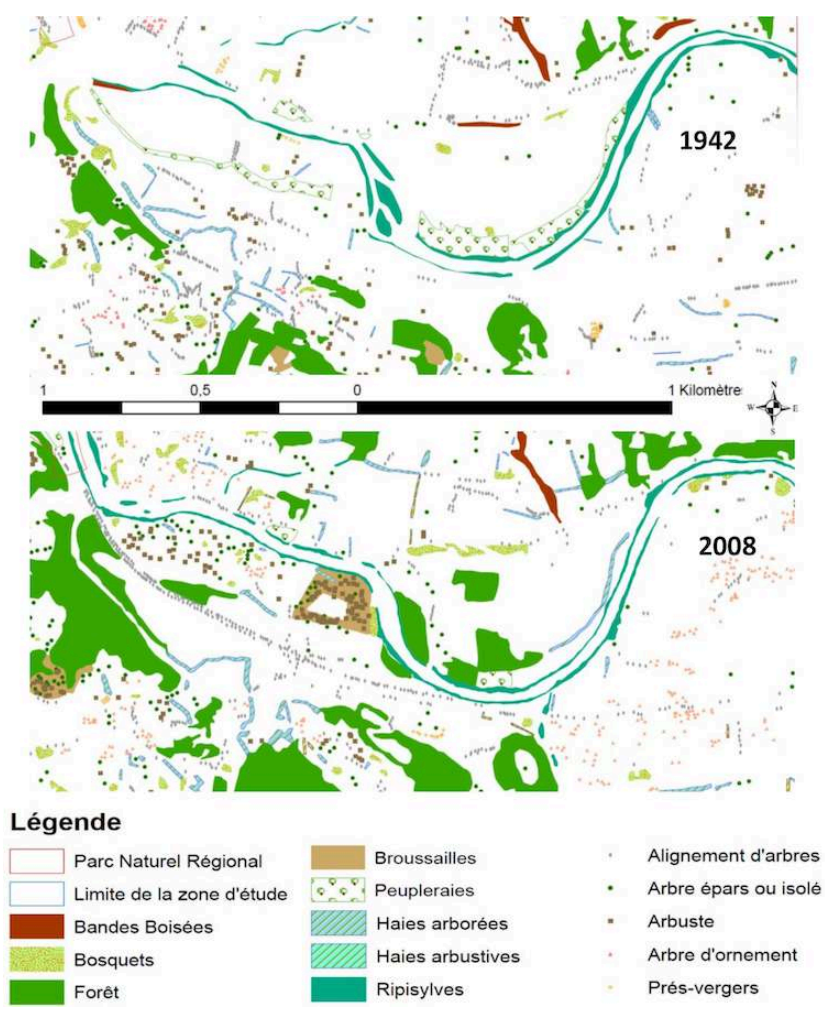

Source : d'après Menillet, 2013.

Parallèlement, si certains des acteurs évoquent un bocage encore présent sur le territoire, la plupart soulignent la forêt qui s'étend: «Il faudrait qu'elle arrête de gagner sur les paysages, c'est bien qu'elle y soit mais il ne faudrait pas qu'elle continue à manger les paysages. » (Un agriculteur.) Cette évolution vers une "uniformisation des paysages ", avec une forêt qui gagne du terrain sur les coteaux et les monocultures qui se sont développées en plaine, inquiète. Selon les acteurs du monde agricole, le paysage doit représenter un "équilibre» entre la nature et les activités humaines, entre les milieux fermés forestiers et les milieux ouverts agricoles. L'un d'eux explique que «c'est peut-être ça qui est le plus attirant dans ce paysage, ici en montagne. Ce subtil équilibre entre les forces de l'humain et les forces de la nature qui se tiennent [...] ». Pour un autre, la beauté des paysages se dégage des prairies entretenues avec des haies, des arbres isolés et la présence de l'élevage. Les acteurs du monde agricole voient l'homme comme un créateur de paysages et plusieurs évoquent également l'idée que l'homme peut améliorer la biodiversité. L'un dit, en parlant cette fois des forêts, qu'il «faut laisser les arbres vieillir et se développer pour que ce soit accueillant pour les espèces animales ».

Les élus abordent la grande diversité des paysages de la basse vallée du Salat, et l'un d'eux la qualifie même "d'un véritable petit paradis vert ». Cependant l'évolution des paysages, caractérisée par l'expansion de la forêt et la régression des prairies, est soulignée, et l'un d'eux (également agriculteur) qualifie les parcelles laissées à l'abandon de "sales». Pour les élus, les agriculteurs sont indispensables pour entretenir les paysages de la vallée et la déprise agricole est une inquiétude et une réelle problématique pour leur avenir et celui de leurs habitants. Néanmoins deux élus 
locaux pensent que les agriculteurs sont indifférents aux paysages et considèrent les problématiques paysagères ou les arbres hors forêt comme secondaires par rapport à leurs objectifs de rendement et aux contraintes que peut engendrer la conservation des arbres hors forêt. Pour autant, ils sont conscients de la difficulté pour les agriculteurs d'entretenir les arbres sur leur exploitation. On retrouve par ailleurs l'argument d'un équilibre entre l'homme et la nature indispensable à la beauté des paysages. La fermeture du paysage par la progression de la forêt et l'enfrichement est soulignée par l'importance de conserver des points de vue sur la chaîne des Pyrénées.

Cet argument est également partagé par les techniciens et chargés de mission pour qui la beauté des paysages de la basse vallée du Salat tient de leur diversité. L'exploitation forestière intensive est perçue comme une menace pour ces derniers. D'une façon générale, les acteurs locaux interrogés souhaitent conserver la beauté et la diversité des paysages de ce territoire grâce à la présence des hommes et de l'agriculture, et la problématique contrastée de l'arbre est importante. Il est considéré à la fois comme une composante essentielle de la beauté des paysages de la vallée et témoin privilégié de l'histoire locale, mais aussi comme une gêne quand le non-entretien des structures arborées lié à la disparition des usages traditionnels conduit à l'enfrichement et au reboisement.

Comme le soulignent Curt et al. (1998), sur des terrains en Auvergne et dans les Causses, arbres et arbustes peuvent constituer la première ossature de la fermeture des paysages en cas de déprise agricole, en particulier en moyenne montagne. Les perceptions diverses de l'arbre dans le paysage de la vallée du Salat tiennent aussi aux différentes fonctions que les acteurs locaux attribuent aux arbres hors forêt. Le nouveau contexte agricole, accompagné d'une spécialisation des productions, a conduit à l'abandon de nombreuses pratiques liées aux AHF, même si des pratiques plus modernes peuvent être mises en exergue (Liagre, 2006). Cependant, leur prégnance dans le paysage de la vallée du Salat tient notamment à une multifonctionnalité en lien avec des aspects économiques, écologiques et patrimoniaux, mettant en évidence atouts, contraintes et enjeux liés à ces arbres, ce qui apparaît également dans la littérature sur d'autres territoires (Pointereau, 2004 ; Guillerme et al., 2009). Après un siècle de forte régression, ces systèmes agroforestiers traditionnels sont au cœur d'actions locales de remise en valeur économique, écologique ou paysagère.

\section{Une valeur économique marginalisée}

19 L'arbre est perçu par les différents acteurs comme un élément naturel de production. Les productions directes concernent en particulier le bois et les fruits. Mais l'arbre hors forêt peut aussi être le symbole et le témoin d'une activité économique aujourd'hui disparue ou en fort déclin : anciens vergers et même reliques de hautains soulignent la place majeure qu'a eue l'arbre hors forêt d'un point de vue économique. Les vergers dans la vallée étaient essentiellement constitués de poiriers, figuiers, pêchers, pommiers, cerisiers et pruniers. Les variétés locales ou variétés anciennes présentaient des capacités de conservation et de résistance aux maladies importantes, qui permettaient aux familles de consommer des fruits frais qui pouvaient être conservés une grande partie de l'année, ou que l'on faisait sécher. La diversité des espèces et des variétés qui composaient les vergers assurait pour chaque famille des fruits frais à différentes saisons. L'excédent de production était vendu à l'extérieur et constituait un 
revenu supplémentaire. Les arbres hors forêt d'une façon générale produisaient du bois de chauffe et du bois de construction (charpentes mais aussi outils, piquets...), fournissaient du fourrage pour le bétail et procuraient de l'ombre aux animaux. Mais il est également souligné que les vergers de plein-vent étaient source de nombreuses activités autres que la récolte ou la transformation des fruits : l'entretien, le greffage et la taille des arbres demandaient de la main-d'œuvre.

Le principal point faible de ces paysages tient au fait que les arbres qui les composent ont perdu à l'heure actuelle l'essentiel de leurs fonctions économiques et leur fondement social (la société a changé, les besoins et attentes de la population actuelle sont totalement différents de celle qui a pu planter la majorité des AHF qui subsistent actuellement). Par exemple, obtenir du bois de chauffage à partir d'une haie demande un entretien sélectif et manuel qui requiert beaucoup de temps et de travail, ce qui nuit à sa rentabilité économique et n'incite guère les agriculteurs à les conserver. Les tentatives de valorisation des haies grâce au bois raméal fragmenté ont encore du mal à émerger. Les projets mis en œuvre et fondés réellement sur les AHF sont encore aujourd'hui essentiellement le fait d'agriculteurs considérés comme «atypiques » ou qui ont le plus souvent d'autres sources de revenus potentiels. Malgré une demande croissante en bois énergie, la dispersion des arbres dans l'espace, qui rend leur exploitation malaisée, ne permet pas encore de revaloriser ce type d'arbres dans ce territoire.

21 L'intérêt de l'arbre hors forêt (bois d'œuvre, bois de chauffe, feuilles fourragères, cidre...) doit constamment être démontré et valorisé sous peine d'abandon, ainsi que toutes les fonctions qu'il peut jouer dans les systèmes de production. Ces usages ne peuvent être dissociés des nombreux savoir-faire allant de la gestion des arbres à la production, à la conservation et à la transformation des produits jusqu'à leur utilisation, voire leur commercialisation.

Différentes associations qui œuvrent à la promotion, à la préservation et à la revalorisation des paysages arborés s'appuient sur la multifonctionnalité des arbres et tentent de redynamiser la fonction productive du patrimoine fruitier. C'est notamment le cas de la fédération Renova, des associations Les Vergers retrouvés du Comminges, Arbres et paysages d'Autan ou Solagro, qui mènent depuis environ vingt ans des actions de sensibilisation, de formation (greffage, conduite de vergers, restauration des vieux fruitiers...) et d'accompagnement pour réintégrer pleinement les variétés anciennes, de pommes notamment, dans l'économie locale. Leurs objectifs sont variés, certaines ne s'intéressent qu'aux vergers traditionnels, tandis que d'autres prennent aussi en compte les pratiques modernes et en devenir, dans le but de :

- maintenir et préserver les AHF existants (par un soutien aux programmes d'entretien et de restauration);

- reconstituer et diversifier les paysages d'arbres hors forêt (PAHF) qui existent (soutien aux programmes de replantation des haies, des bosquets...);

- implanter des AHF dans des espaces qui en sont dépourvus (soutien aux planteurs agroforestiers).

Les associations régionales et locales ne sont pas toutes très actives, mais la création des deux associations Association française d'agroforesterie (AFAF) et Association française arbres champêtres et agroforesteries (AFAC-Agroforesteries) a impulsé un élan au niveau national. 

peut aussi citer les Plans de développement ruraux (PDR), qui intègrent sans les nommer les paysages d'arbres hors forêt. Mais les mesures sont généralement ambiguës, mélangeant patrimoine rural et valeurs de la "nature", et se référant rarement au concept de paysage. À cet égard, la prise de conscience par les pouvoirs publics des avantages de l'agroforesterie (au sens large), et de la possibilité d'associer des arbres et des cultures dans des conditions d'exploitations modernes, se manifeste par une évolution favorable des critères d'éligibilité aux aides de la politique agricole commune (PAC). Ces mesures, bien que limitées, sont considérées par les agriculteurs comme une amorce de réponse à leurs besoins. Les mesures envisagées au niveau de la PAC mettent en évidence un problème fondamental : l'intérêt pour la protection de ce patrimoine n'est pas tant lié aux fonctions agricoles, pastorales ou forestières, mais plus à l'idée de préservation des valeurs intrinsèques et naturelles de pratiques agricoles traditionnelles perçues parfois comme folkloriques par certains acteurs à d'autres échelles territoriales. Cependant, le dynamisme récent autour des formes modernes d'agroforesterie pourrait à terme profiter aussi aux paysages dits «traditionnels».

D'un point de vue technique, certains acteurs soulignent qu'il serait nécessaire que les pratiques anciennes puissent être modernisées pour s'adapter aux contraintes actuelles (afin d'éviter par exemple que l'élagage soit un travail trop compliqué). Il faut surtout $\mathrm{du}$ temps pour convaincre les propriétaires et les agriculteurs de (re)planter. Le contexte social, notamment le vieillissement de la population, contribue à rendre cette redynamisation difficile car les agriculteurs en activité appartiennent pour beaucoup à une génération qui a été formée en faveur de la grande mécanisation et des remembrements. Enfin, la question de la formation semble être un enjeu majeur, quels que soient les acteurs concernés. Le manque de formation spécifique des futurs agriculteurs, techniciens, conseillers, animateurs... est problématique pour une prise en compte des AHF et des paysages dans leur métier à venir. 


\section{Une valeur écologique qui s'affirme}

28 La littérature abonde désormais de références qui soulignent les avantages écologiques liés aux arbres hors forêt, et remplissent de nombreux services écosystémiques. Haies et ripisylves permettent par exemple la filtration de l'eau et constituent une protection contre l'érosion des sols et les incendies (Picard, 1996). Elles fixent également les nitrates et limitent la vulnérabilité des sols aux intrants agricoles comme les pesticides (Macary et Bordenave, 2008), et permettent d'assurer la connexion entre les entités naturelles, comme les ensembles forestiers, par la fonction de corridors biologiques et de lieu de conservation de la biodiversité. L'abri et la ressource alimentaire assurés par les arbres et les arbustes, ainsi que leur fonction de corridor écologique favorisent les populations d'animaux sauvages, en particulier les petits mammifères (Butet et al., 2006). La protection des insectes pollinisateurs (syrphes, bourdons, abeilles sauvages et domestiques...) et des prédateurs de parasites (carabes, coccinelles, etc.) assurée par le couvert végétal associé à l'arbre hors forêt contribue en outre à l'amélioration de la production agricole et à sa protection (Marshall, 2004). Les haies fournissent aussi l'abri et le couvert pour les pollinisateurs, eux-mêmes indispensables pour les vergers mais également pour l'agriculture en général (Vaissière et al., 2008 ; Maire et Laffly, 2015). La prise de conscience de la perte de cette biodiversité a tout d'abord été le fait de particuliers et du réseau associatif (Marchenay, 1987).

L'ensemble des acteurs locaux rencontrés sur le territoire du Salat citent les mêmes avantages et services écosystémiques des arbres hors forêt que ceux abordés dans la littérature. Les personnes enquêtées évoquent l'ombre que les arbres procurent aux hommes et aux animaux. Elles parlent aussi de l'effet brise-vent des arbres qui peut protéger des bâtiments et des cultures, ainsi que son effet contre l'érosion des sols qui ne représente pas un enjeu majeur sur ce territoire selon les acteurs. La fonction écologique de l'arbre est importante pour la majorité des personnes interrogées : c'est une niche écologique pour les animaux, il a un rôle d'épuration des eaux polluées et des intrants chimiques, il enrichit et fertilise le sol et augmente sa biodiversité, il régule la température et dynamise la vie de manière générale. La présence des arbres hors forêt est perçue comme offrant un refuge pour les animaux et les auxiliaires de culture. Cet effet refuge est en revanche parfois perçu de façon négative, notamment par nombre d'agriculteurs, car l'enfrichement favorise le développement d'espèces considérées comme nuisibles, tel le sanglier.

Certains acteurs restent centrés sur leur spécialité, leur champ d'action ou leur expérience. Un élu insiste sur le rôle de protection de l'arbre contre les inondations et un autre met en avant son action contre le ruissellement des eaux. Une spécialiste de l'environnement qui s'occupe des berges du Salat insiste sur les services écosystémiques rendus par les ripisylves (maintien des berges ou épuration du cours d'eau). Les techniciens agricoles énoncent le paillage pour les litières animales, l'épandage sur les parcelles agricoles et les différents services écosystémiques de l'arbre hors forêt bénéfiques à l'agriculture.

31 Des inconvénients sont cependant abordés, comme la gêne que les arbres représentent pour le passage de l'eau en créant des embâcles, raison pour laquelle les communes décident d'enlever les arbres morts le long des cours d'eau. Une autre spécialiste de l'environnement parle aussi des arbres qui colonisent les prés et du risque d'incendie que cela représente. La plupart des associations, ainsi que les chargés de mission, 
insistent cependant sur l'importance des services écosystémiques liés à l'arbre, élément qui se révèle essentiel au bon état de la biodiversité terrestre et participe à la limitation des risques naturels.

En lien avec la biodiversité et l'environnement, le paysage arboré et sa valorisation apparaissent pour la majorité des acteurs locaux comme un levier pour la mise en place de la politique de la trame verte et bleue (TVB). Dans ce dispositif, l'arbre hors forêt devient un élément des continuités écologiques permettant de protéger la biodiversité et faisant partie intégrante de la gestion d'un territoire dans le cadre d'une politique de développement durable. Il faut cependant noter que la trame verte et bleue a rarement été citée directement comme un enjeu du territoire par les acteurs interrogés, notamment parce que la majorité des enquêtés ne connaît pas cette proposition environnementale. Certaines personnes, qui déclaraient ne pas connaître ce dispositif, en avaient pourtant une opinion favorable (théoriquement du moins, après explication), ce qui représente un paradoxe à souligner. Au contraire la majorité des agriculteurs y était plutôt hostile. Pour autant, l'ensemble des acteurs locaux est sensible à la problématique de la perte de la biodiversité comme nous l'avons mentionné, raison d'être de la trame verte et bleue.

La promotion récente du discours environnementaliste, qui fait écho aux inquiétudes sociétales et aux exigences incantatoires du développement durable, a entraîné un regain d'intérêt pour les paysages arborés et leurs productions. Le développement durable et le paysage sont invoqués pour initier et soutenir les tentatives de redynamisation de ces espaces délaissés et de ces formations arborées en voie d'abandon. À défaut d'avoir des pratiques sources de rentabilité économique, le discours se fonde avant tout sur les dimensions sociales et écologiques pour recréer ou entretenir ces paysages.

Cette dimension écologique est d'ailleurs également présente à l'échelle nationale, et peut contribuer au renouveau des paysages de l'arbre hors forêt. C'est la dimension qui apparaitt le plus dans les discours, au point de faire oublier que la fonction première de ces paysages de polyculture arborée était la production de biens alimentaires et de bois.

\section{Une valeur esthétique, patrimoniale et identitaire importante}

Quand on parle des avantages de l'arbre hors forêt, les différents acteurs invoquent leur valeur esthétique dans les paysages. Les acteurs locaux sont sensibles à la beauté de l'arbre hors forêt mais également à ce qu'il représente. Pour de nombreux élus notamment, l'arbre hors forêt devient une préoccupation afin de préserver une qualité paysagère et un cadre de vie agréables et attractifs. Bon nombre des entretiens montrent que la composition des paysages de la basse vallée du Salat, avec la diversité des espèces et des variétés d'arbres, de leurs fonctions, de leurs modes de gestion et de leurs utilisations, a contribué à renforcer le sentiment d'appartenance à ce territoire. Le paysage arboré est d'ailleurs évoqué par certains comme un patrimoine, témoin de l'histoire locale.

Certains agriculteurs entretiennent une relation particulièrement intime avec les arbres, l'un d'eux expliquant: «[...] C'est vrai que les arbres c'est toute une histoire, c'est rassurant. Tu sais qu'ils étaient là avant toi et qu'ils y seront après. C'est toute une 
génération, et puis c'est une présence qui est enracinée, qui est présente. » Mais les acteurs ne sont pas tous sensibles à ce patrimoine, et de vieux arbres sont laissés à l'abandon et dépérissent. Une situation assez généralisée sur le Comminges et le Couserans est l'arrachage des arbres par les héritiers. Tant que l'« ancien » est encore en vie et accorde de l'importance aux vergers et autres arbres hors forêt, ceux-ci sont maintenus. Mais quand il disparaît, les héritiers, surtout lorsqu'ils vivent au loin, ne conservent pas forcément ces arbres, pour des raisons foncières ou pratiques (contraintes de travail, manque de temps et de moyens à accorder à l'entretien des arbres). Parfois au contraire ces arbres sont maintenus en mémoire des personnes disparues, et surtout pour la beauté des paysages à laquelle ils participent. Ces différentes considérations contribuent à créer et à entretenir (ou non) un sentiment d'attachement pour ces paysages.

Certains acteurs expliquent également que dès leur plus jeune âge, ils avaient observé leurs grands-parents et leurs parents qui effectuaient les travaux de greffage et d'entretien des arbres. Ils avaient appris avec eux les spécificités des différentes variétés, et les méthodes de transformation des fruits... Aujourd'hui, les personnes qui étaient en capacité de transmettre les connaissances et les savoir-faire sur les arbres et l'arboriculture fruitière traditionnelle disparaissent, ou sont trop âgées pour continuer à le faire. Avec elles, c'est aussi une partie de cette culture qui s'efface, malgré la volonté de certains acteurs de la maintenir et de la renouveler.

Cependant, les décennies 1970-1990 ont été marquées par la venue sur le territoire pyrénéen de personnes à la recherche d'un nouveau cadre de vie. Souvent arrivées d'autres régions et plus généralement de la ville, elles aspiraient à un mode de vie différent, plus proche de la nature. Ces personnes, sensibles à ces paysages arborés, sont arrivées avec un nouveau regard sur la campagne qui a notamment contribué au renouveau d'anciens vergers par des actions de sauvegarde active du patrimoine fruitier traditionnel.

La distinction est nette entre des éléments arborés témoins de pratiques passées, et des arbres «sauvages » qui contribuent à la fermeture des paysages du fait de l'expansion de la forêt, phénomène reconnu par tous les acteurs interrogés comme la problématique prioritaire sur ce territoire, en lien avec la déprise agricole. Ces enjeux paysagers sont aussi en lien avec l'objectif économique d'attirer une population touristique. Les élus du territoire de la basse vallée du Salat sont conscients de l'intérêt de protéger ces espaces naturels et la qualité de leurs paysages pour dynamiser un tourisme vert qui contribuerait à développer l'économie de la vallée. Ce tourisme s'appuie déjà sur le patrimoine rural de la basse vallée du Salat autant architectural que naturel, dont la valorisation augmente l'attractivité du territoire. Le développement du patrimoine rural passe également par la valorisation de savoir-faire et la promotion de produits locaux, mais aussi par la mise en valeur des arbres hors forêt. Les vergers anciens en particulier portent une valeur patrimoniale forte qui s'inscrit aisément dans une démarche touristique. Les fonctions paysagères et culturelles qu'ils assurent sont relayées par une fonction de loisir (lieu de promenade, d'inspiration, de repos). De ce point de vue, ces paysages sont pris en compte dans les stratégies d'aménagement du territoire de la basse vallée du Salat.

40 Ainsi les considérations de certains porteurs de projets à l'échelle territoriale ne sont pas forcément en phase avec les attentes et besoins exprimés par les réels "producteurs» des paysages d'arbres hors forêt que sont - majoritairement - les 
agriculteurs. Pour ces derniers, c'est avant tout l'aspect productif et économique qui prime. En revanche, ils n'hésitent pas à assurer la promotion de leurs produits en valorisant la notion de paysage (qualité paysagère où l'arbre est mis en valeur). D'une façon générale, la prise de conscience de l'impact paysager des pratiques agricoles a malgré tout, pour le moment, plus d'importance aux yeux des néoruraux et des nonagriculteurs, qu'à ceux des exploitants. La notion de paysage n'est pas appréhendée comme une contrainte par les "producteurs de paysage ", ni même comme un objectif important (sur le territoire étudié, mais également dans d'autres espaces de moyenne montagne).

\section{Conclusion}

41 L'arbre structure le paysage, permet de le décrire et parfois de comprendre les choix des aménagements forestiers et ruraux pluriséculaires d'une société (Galochet et Simon, 2003 ; Mansion, 2010). Objet de nature pour les uns, élément de l'agrosystème pour d'autres, lieu de culte et de vénération, ou bien encore objet esthétique et artistique, l'arbre hors forêt est à l'origine d'une grande diversité de perceptions et de représentations paysagères, individuelles et collectives, qu'elles soient visuelles, littéraires, picturales, photographiques ou même cinématographiques (Mottet, 2002). Il tient souvent une place importante dans l'imaginaire populaire local. En fonction du contexte socioculturel local ou régional, l'arbre hors forêt a une valeur symbolique, parfois légendaire, mythologique ou religieuse, évoquant la mémoire d'un lieu (Ubaud, 1997). Les arbres hors forêt sont bien connus des agriculteurs, mal cernés par les gestionnaires et peu pris en compte dans les statistiques officielles et les aides au développement (Bellefontaine et al., 2001).

42 La basse vallée du Salat, qui a servi de terrain d'étude, peut être considérée comme un "paysage rural ordinaire", ni périurbain ni montagnard isolé. C'est un territoire où l'arbre a toujours fortement structuré le paysage, que ce soit par l'abondance des forêts que par sa présence dans les terres agricoles. Aujourd'hui, les phénomènes parallèles de progression des boisements du fait de la déprise agraire, d'une part, et de l'élimination des AHF dans les terroirs, pour des raisons de modernisation, d'autre part, rompent les équilibres anciens. Le travail d'enquête mené pour cette étude a permis de mettre en évidence une double valeur accordée aux arbres hors forêt.

Les acteurs institutionnels ont tendance à privilégier la valeur environnementale mais, au-delà du bénéfice tiré de ces fonctions, ces paysages sont présentés comme le reflet de l'histoire du territoire, porteurs d'une dimension culturelle. Cette valeur culturelle des arbres apparait comme un atout majeur pour les différents acteurs; les habitants et les agriculteurs, notamment, sont attachés aux arbres, parfois par nostalgie. Cependant, le patrimoine des AHF ne doit pas uniquement être considéré comme une valeur qui permet d'arbitrer des mesures pour sa conservation et sa restauration, mais comme ayant des potentialités économiques indéniables. La multifonctionnalité de ces paysages apparaît comme un atout, et les productions issues de ces systèmes sont réputées pour leur qualité. Or, le principal point faible de ces paysages tient au fait que les arbres qui les composent ont perdu à l'heure actuelle l'essentiel de leurs fonctions économiques et de leur fondement social (les besoins et attentes de la population actuelle sont totalement différents de celle qui a planté la majorité des AHF qui subsistent actuellement). Il est nécessaire de faire prendre conscience aux responsables 
et aux fonctionnaires des services agricoles et environnementaux de la nécessité de considérer davantage ces paysages en tant que fournisseurs de services écosystémiques mais qui risquent de disparaitre alors qu'ils caractérisent encore largement l'espace rural actuel.

Ce constat est largement transposable à l'ensemble de l'aire géographique européenne à cause de l'importance historique de l'héritage des savoirs locaux encore prégnants, notamment agricoles. Le paysage, en ce qui concerne les AHF, est généralement revendiqué par tous, mais sa prise en compte est encore faible dans les faits. Une approche territoriale des paysages d'arbres hors forêt, intégrant leur diversité, leur dimension historique et les pratiques qui leur sont liées, est nécessaire. Mais il serait intéressant d'aborder aussi le paysage, du fait de la polysémie de ce terme, comme un paradigme de médiation entre le territoire et la société qui l'habite, et de comprendre comment les paysages d'arbres hors forêt participent d'un sentiment d'appartenance à un territoire quand ils ne sont pas répertoriés comme remarquables, malgré une variété paysagère singulière et typée. Il s'agirait, à travers l'objet " arbre hors forêt " d'analyser le rôle du paysage comme support et vecteur d'une identité collective et individuelle. La sensibilisation des différents acteurs à l'importance de l'arbre dans le paysage rural passe par une action simultanée aux différentes échelles territoriales. Pour cela, l'identification des dynamiques historiques est une étape importante pour l'intégration des PAHF dans des plans d'aménagement spécifiques. Si l'on considère l'objectif de préservation des PAHF (et plus généralement les patrimoines historicoenvironnementaux) comme un problème de "conservation active ", il est possible de formuler des propositions qui renforcent la dimension locale de la gestion, reconnaissant le rôle des producteurs locaux également dans la conservation des paysages culturels.

Nous tenons à remercier le ministère en charge de l'Environnement qui, grâce aux programmes "Paysages et développement durable 1 et 2 ", a soutenu les recherches que nous avons menées sur les paysages d'arbres hors forêt. Nous remercions également tous les acteurs locaux qui ont prêté leur concours à ces travaux.

\section{BIBLIOGRAPHIE}

Bellefontaine, R., Petit, S., Pain-Orcet, M., Deleporte, P., \& Bertault, J. G., Les Arbres hors forêt. Vers une meilleure prise en compte, Rome, FAO (Cahiers FAO conservation $\mathrm{n}^{\circ} 35$ ), 2001, $231 \mathrm{p}$.

Butet, A., Paillat, G., Delettre, Y., "Factors driving small rodents assemblages from field boundaries in agricultural landscapes of western France", Landscape Ecology, 2006, 21(3), p. 449-461.

Conseil de l'Europe, Convention européenne du paysage et documents de référence, Strasbourg, Éditions du Conseil de l'Europe, 2000, 98 p.

Coulon, F., Pointereau, P. et al., Le Guide technique du pré-verger pour une agriculture durable, Toulouse, Solagro, 2005. 
Coulon, F., Meiffren, I. et al., Architectures végétales de Midi-Pyrénées : Haies, arbres et vergers, Toulouse, Solagro, 2003.

Coulon, F., Dupraz, C., Liagre, F., Pointereau, P., «Étude des pratiques agroforestières associant des arbres fruitiers de haute-tige à des cultures et pâtures », rapport au ministère de l'Environnement, Solagro, 2000.

Curt, T., Prevosto, B., Marsteau, C., Michalland, B., Loiseau, B., Loubier, K., « Typologie et gestion des accrus de moyenne montagne », rapport, GECOTER/ECOFRICH, 1998, 113 p.

Galochet, M., Simon, L., L'Arbre du géographe : un objet entre nature et société, Paris, L'Harmattan, coll. « Kubaba », série « Actes II », 2003, p. 29-49.

Guillerme, S., «Interface. Dynamiques paysagères et perception des interfaces arborées. Quels enjeux pour la mise en place de la trame verte et bleue? ", rapport final du programme « Paysage et développement durable 2 (PDD2, MEDDE) », 2014, 103 p.

Guillerme, S., Jimenez, Y., Moreno, D., « Les paysages d'arbres hors forêt, des paysages porteurs des enjeux du développement durable », dans Luginbhül, Y. et Terrasson, D., Paysage et Développement durable, Versailles, Éditions Quae, coll. « Update Sciences et technologies », 2013, p. 37-47.

Guillerme, S. (dir.), « Les paysages d'arbres hors forêt (PAHF). Multi-valorisation dans le cadre d'un développement local durable en Europe du Sud », rapport final de recherche dans le cadre du programme PDD (MEEDAT), GEODE-CNRS, Toulouse, 2010, $282 \mathrm{p}$.

Guillerme, S., Alet, B. et al., « L'arbre hors forêt en France. Diversité, usages et perspectives », Revue Forestière Française. Les nouveaux usages de l'arbre, nº 5, 2009, p. 543-560.

Liagre, F., Les Haies rurales : rôles, création, entretien, Paris, Éditions France Agricole, 2006, 319 p.

Luginbuhl, Y., « Programme de recherche Politiques publiques et paysages : analyse, évaluation, comparaison, synthèse des résultats scientifiques ", ministère de l'Écologie et du Développement durable, Cemagref, 2004, 22 p.

Macary, F., Bordenave P., De Ville D’Avray N., « Estimation d'un risque environnemental : la contamination des eaux de surface par les intrants agricoles. Application sur les coteaux de Gascogne », colloque « Vulnérabilités sociétales, risques et environnement : comprendre et évaluer », Toulouse, Ruramen/Ceres, 2008.

Maire, E., Marais-Sicre, C., Guillerme, S., Rhoné, F., Dejoux, J.-F., Dedieu, G., « Télédétection de la trame verte arborée en haute résolution par morphologie mathématique ", Revue internationale de Géomatique, vol. 22, $\mathrm{n}^{\circ}$ 4, 2012, p. 519-538.

Maire, E., Laffly, D., Abeilles et paysages. Enjeux apicoles et agricoles, Versailles, Éditions Quae, 2015. Mansion, D., Les Trognes, l'arbre paysan aux mille usages, Rennes, Éditions Ouest-France, 2010, 143 p. Marchenay, P., Lagarde, M. F., À la recherche des variétés locales de plantes cultivées, Hyères/Paris, PAGE-PACA, Bureau des ressources génétiques, 1987.

Marshall, E. J. P., « Agricultural landscapes: field margin habitats and their interaction with crop production ", Journal of Crop Improvement, 12(1/2), 2004, p. 365-404.

Menillet, P.-H., « Évolution de la trame verte dans la basse vallée du Salat. Apport des SIG dans la cartographie de la trame verte arborée en lien avec la biodiversité », mémoire de master 2 géographie et aménagement, université de Toulouse Jean-Jaurès, 2013. 
Mottet, J. (dir.), L'Arbre dans le paysage, Seyssel, Éditions Champ Vallon, coll. « Pays-paysages », $2002,280 \mathrm{p}$.

O'Rourke, E., Kramm, N., « High nature value (HNV) farming and the management of upland diversity, A review », European countryside, vol. 4, n² 2, 2012, p. 116-133.

Picard, O., « Scénarios technico-économiques de boisement de parcelles d'exploitations agricoles en Midi-Pyrénées ", dans Balent, G., La Forêt paysanne dans l'espace rural. Biodiversité, paysages, produits, Paris, Inra, coll. « Études et recherches sur les systèmes agraires et le développement ", 1996, p. 213-217.

Pointereau P., " Systèmes agroforestiers et bocagers, savoirs locaux et biodiversité », dans Bérard, L., Cegarra, M., Djama, M. et al., Biodiversité et Savoirs naturalistes locaux en France, Paris, Cirad, Iddri, IFB, Inra, 2005, p. 119-126.

Pointereau, P., « L'arbre et le paysage : enjeux environnementaux et reconnaissance de la multifonctionnalité », Comptes rendus de l'Académie d'agriculture de France, nº 90, 2004.

Sheeren, D., Bastin, N., Ouin, A., Ladet, S., Balent, G., Lacombe, J.-P., "Discriminating small wooded elements in rural landscape from aerial photography: a hybrid pixel/object-based analysis approach", International Journal of Remote sensing, vol. 30, nº 19, 2009, p. 4979-4 990.

Ubaud, J., Des arbres et des hommes, architecture et marqueurs végétaux en Provence et Languedoc, Aix en Provence, Edisud, 1997.

Vaissière, B., Gallai, N., Carré, G., Bommarco, R., Krewenka, K., Morison, N., Szentgyörgyi, H »., " Assessing the potential impact of declining insect pollination service to crops in Europe ", 3rd European Conference of Apidology. 2008-09-08 to 2008-09-11, Belfast, GBR, Queen's University, 2008.

\section{RÉSUMÉS}

Héritages de système s ruraux qui ont utilisé l'arbre comme élément structurant, les paysages d'arbres hors forêt sont encore aujourd'hui en France un patrimoine menacé et peu pris en compte dans les politiques publiques. Pourtant, ils représentent un enjeu environnemental et politique du fait des problèmes et des défis qu'ils posent, mais aussi par leurs potentialités, notamment en termes de services écosystémiques. Cette arboriculture a historiquement contribué à la diversité végétale ou paysagère des territoires. En tant que composante de systèmes agraires très anciens, l'arbre hors forêt s'inscrit dans la durée. Ces paysages, vecteurs de biodiversité, représentent aussi une dimension du patrimoine culturel prise en compte dans les politiques de développement locales et territoriales. Centré sur le département de l'Ariège, dans un contexte de fermeture des milieux, l'article aborde la dynamique et la perception des paysages d'arbres hors forêt, la place de ces paysages dans les politiques publiques et les projets de développement territorial, ainsi que les enjeux liés à la conservation ou à la valorisation de systèmes arborés économiquement et écologiquement marginalisés.

As the heritage of rural systems which have used the tree as a structuring element, landscapes with non-woodland trees are under threat and are rarely taken into consideration in public policies. Yet they are of environmental and political importance not only due to the problems and challenges they present but also because of their potential, namely in terms of ecosystemic services. This type of tree cultivation historically contributed to plant and landscape diversity. The non-woodland tree has always been a component of very ancient agrarian systems. These landscapes which ensure biodiversity, also represent a cultural heritage dimension taken into account in local development policies. Focusing on the département of Ariège in the context of the 
closure of nature areas, the article addresses the dynamics and representations of landscapes with non-woodland trees, the role of these landscapes in public policies and regional development projects, as well as challenges regarding the conservation and valorisation of tree systems economically and ecologically marginalised.

\section{INDEX}

Keywords : non-woodland trees, representations, heritage, landscape dynamics, multi-valuation Mots-clés : arbre hors forêt, perceptions, patrimoine, dynamique paysagère, multivalorisation

\section{AUTEURS}

\section{SYLVIE GUILLERME}

Elle est chargée de recherche au CNRS. Ses travaux portent sur une approche géographique des dynamiques paysagères et de la gestion de la biodiversité dans les espaces naturels et ruraux, en s'intéressant plus particulièrement aux activités humaines génératrices de biodiversité, notamment en matière de gestion, pratiques et représentations des arbres hors forêt. sylvie.guillerme[at]univ-tlse2[dot]fr

\section{JEAN-PAUL MÉTAILIÉ}

Il est directeur de recherche au CNRS. Ses travaux portent sur les relations sociétésenvironnement, l'histoire de l'environnement ; les sources iconographiques, photographiques et les observatoires des paysages ; les pratiques et la construction des paysages (le feu, le brûlage dirigé) et la gestion environnementale. jean-paul.metailie[at]univ-tlse2[dot]fr

\section{ÉRIC MAIRE}

Ingénieur de recherche au CNRS, il consacre ses travaux aux dynamiques et aux enjeux contemporains des environnements et des paysages liés à la biodiversité, à l'agroforesterie, au pastoralisme et à l'apiculture en lien avec les politiques publiques. Par ailleurs, il met en œuvre des outils de spatialisation, de télédétection, d'analyse d'images en haute résolution dédiés aux recherches sur les sociétés pour la préservation des espaces protégés et de ses marges. eric.maire[at]univ-tlse2[dot]fr 